\title{
Lunar probe ready to bite the dust
}

After nearly three years in space, Europe's Moon mission is out of fuel. On 3 September the spacecraft SMART-1 will smash into the lunar surface near the Lake of Excellence in the Moon's mid-southern latitudes. Astronomers hope the dust kicked up from the rocky site will provide information about the Moon's composition and impact history.

Researchers at the European Space Agency (ESA) are very happy with what the mission has achieved. The craft was the agency's testbed for a new type of thruster called an ion engine. SMART-1 is the second such engine to be used in space - the first was on board NASA's comet-chaser Deep Space 1.

Ion engines work by accelerating a stream of ionized atoms through an electric field. The one on SMART-1 provides about as much force as a postcard resting in the palm of your hand. That made its journey painfully slow: it took 14 months and a roundabout trip of 84 million kilometres for it to spiral out of Earth's orbit and reach the Moon. In contrast, Apollo 11's conventional propellants allowed it to travel a roughly 400,000-kilometre line between Earth and the Moon in just over 3 days.

The upside is that SMART-1's journey used around $70 \mathrm{kilograms}$ of fuel, about ten times less than would have been needed with regular propellants. So, although ion engines pick up speed quite slowly, they are extremely effective - and fast - for long-distance trips using light craft.

Learning to drive an ion engine is different from using chemical propellants, according to SMART-1 project scientist Bernard Foing. Chemical propellants are used in short burns lasting seconds or minutes. For those critical periods a craft must be precisely controlled, but otherwise it coasts passively to its destination. But ion drives are always on, so the course of the craft must be constantly monitored and corrected. "It's much more complicated than driving with chemical energy," says Foing.

Despite a few episodes in

"Using an ion engine is much more complicated than driving with chemical energy." ously switched itself off, Foing says that SMART-1 performed well - good news for ESA's upcoming BepiColombo mission to Mercury in 2013. BepiColombo will use conventional propellants to break out of Earth's orbit quickly, then an ion drive to speed towards the Sun. The engine's tiny but continuous thrust over the course of months should cut flight time in half.

Once SMART-1 arrived at its destination, it used a digital camera and two spectrometers to

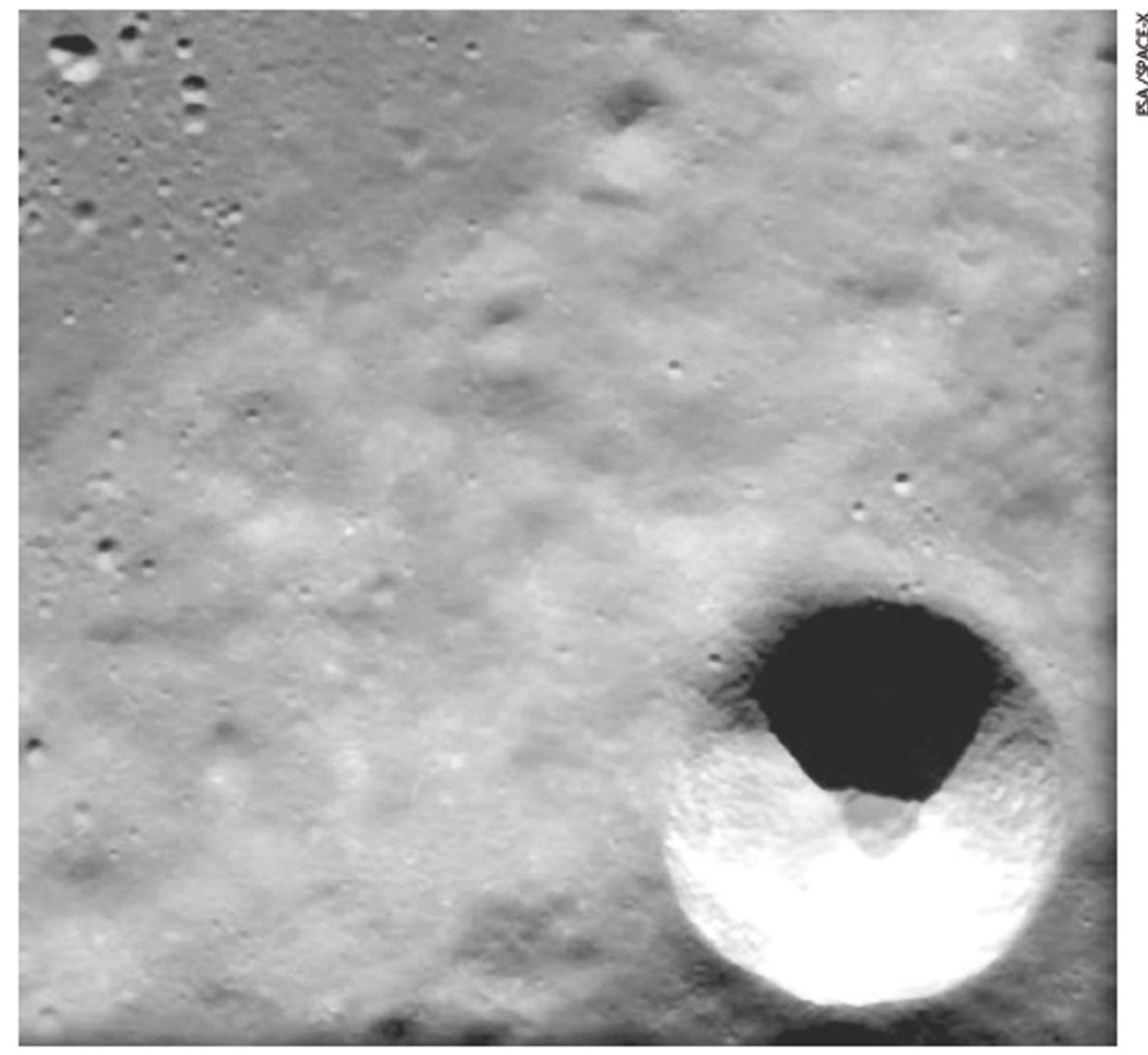

Moonshot Europe's SMART-1 probe has offered astronomers a fresh view of the lunar surface.

survey the Moon's surface. The craft orbited for 22 months gathering data on the abundances of calcium, aluminium, magnesium and silicon on the lunar surface.

Several teams have been awaiting the release of the full results. For example, Wim van Westrenen, a petrologist at the Free University in Amsterdam, hopes they will help him investigate the Moon's origin. Most lunar researchers believe that the satellite formed when another body crashed into Earth billions of years ago. But it's not dear, for example, which of the two colliding bodies contributed most of the Moon's mass.

${ }^{\alpha}$ There are dozens of models for where and how and what materials melted in the Moon," says van Westrenen. In a five-year project starting in January 2007, he hopes to work out how the process occurred by crushing various combinations of materials at the high temperatures and pressures that would have been present when the Moon formed, to try to recreate the exact composition detected by SMART-1.

ESA's mission will be followed by a glut of lunar visits. In 2007, Japan is scheduled to launch SELENE, a 3-tonne spacecraft that will survey the Moon's mineralogy, topology and gravity gradients. The same year, India will launch Chandrayaan-1 and China will launch Chang'e 1 - both will be those countries' first missions beyond Earth orbit. In 2008 NASA will launch its Lunar Reconnaissance Orbiter to survey the Moon's surface.

The overlap probably has more to do with national pride than science. But Paul Spudis, a lunar specialist at Johns Hopkins University's Applied Physics Laboratory in Laurel, Maryland, says that the data will be welcome nonetheless. In the rush to beat the Soviet Union to the Moon, the Apollo programme did only piecemeal mapping of the satellite's surface. And after astronauts finished their visits, he says it was ignored for decades by planetary researchers. "Mars has better global imaging than the Moon does."

Geoff Brumfiel 